\title{
Difference in antigenic determinant profiles between human and rat myeloperoxidase
}

\author{
Y. C. PATRY $\dagger$, P. H. NACHMAN§, M. A. P. AUDRAIN*, R. J. FALK§, K. MEFLAH $\dagger \&$ V. L. M. ESNAULT $\ddagger$ Departments of \\ *Immunology and $\$$ Nephrology and Clinical Immunology, Nantes University Hospital and $\dagger$ INSERM U419, Nantes, France and \\ $\S$ Department of Nephrology, University of North Carolina, School of Medicine, Chapel Hill, NC, USA
}

(Accepted for publication 3 April 2003)

\begin{abstract}
SUMMARY
We tested whether rat and human MPO have similar antigenic determinants using 36 human MPOANCA positive sera, one mouse anti-rat MPO and four mouse anti-human MPO monoclonal reagents. Purified rat and human MPO were used in ELISA, with or without crossinhibition by preincubation with human MPO or irrelevant antigen in the liquid phase. Only one human MPO ANCA positive serum exhibited significant binding in rat MPO ELISA. This binding was poorly inhibited by preincubation with human MPO in the liquid phase, but was conserved after adsorption of non specific anti-rat activity in a chromatography column. Three mouse anti-human MPO IgG monoclonal antibodies did not recognize rat MPO. Only one mouse anti-human MPO IgA monoclonal antibody bound to rat MPO. This binding was poorly inhibited by preincubation with human MPO (35\% at $2 \mu \mathrm{g} / \mathrm{ml})$. Conversely, the mouse anti-rat MPO monoclonal did not bind human MPO. We have concluded that: (1) Most human MPO-ANCA recognize antigenic determinants on human MPO which are absent on rat MPO. Therefore, human auto-antibodies bind to epitopes which recently appeared after species evolution; (2) Inversely, the mouse anti-rat MPO monoclonal do not bind human MPO. Therefore, rat MPO epitopes have been altered during species evolution; (3) Mice injected with human MPO preferentially develop antibodies against xeno-epitopes which are not present in rodents. Therefore, human MPO may not be the best antigen to raise ANCA in animal models and (4) A comparison of the amino acid sequences of rat and human MPO may help elucidate the major antigenic epitopes.
\end{abstract}

Keywords ANCA myeloperoxidase epitope species evolution monoclonal antibody

\section{INTRODUCTION}

Anti-neutrophil cytoplasmic antibodies (ANCA) are found in small vessel vasculitides, namely Wegener's granulomatosis and microscopic polyangiitis. These autoantibodies are directed against proteins contained in polymorphonuclear (PMN) granules and monocytes lysosomes, mainly proteinase-3 (PR-3), a serine protease, and myeloperoxidase (MPO), an enzyme involved in the production of reactive oxygen intermediates [1]. ANCA may not be only markers of disease activity [2], but could play a direct role in the pathophysiology of systemic vasculitis. After priming neutrophils with a pro-inflammatory cytokine $\mathrm{TNF} \alpha, \mathrm{PR} 3$ and MPO are expressed at the cell surface, and

Correspondence: Prof Vincent L.M. Esnault, Service de NéphrologieImmunologie Clinique, Hôtel Dieu, 30 bd. Jean Monnet, 44093 Nantes, France.

E-mail: vesnault@nantes.inserm.fr

2003 Blackwell Publishing Ltd subsequent binding of ANCA induce a respiratory burst as well as degranulation of proteolytic enzymes [3], that can lead to lysis of endothelial cell cultures [4].

ANCA can be generated in animal models using various strategies. MPO-ANCA was first induced by $\mathrm{HgCl}_{2}$ injection in Brown Norway (BN) rats that exhibited gut vasculitis [5]. In several other studies, BN rats were immunized with human MPO, and inflammation was targeted in the kidney by MPO and $\mathrm{H}_{2} \mathrm{O}_{2}$ infusion [6], ischaemia [7], or a subnephritogenic dose of antiglomerular basement membrane antibodies [8]. The lung was also successfully targeted using similar strategies [9]. However, the relevance of xeno-antigen injections to induce anti-MPO antibodies in rats remains debated since novel antigen determinants may have appeared during species evolution.

The aim of this study was to test the hypothesis that rat and human MPO may exhibit different epitope profiles, using both purified rat and human MPO, human MPO-ANCA, and mouse anti-human and rat MPO monoclonal reagents. 


\section{MATERIALS AND METHODS}

\section{Rat MPO purification}

Rats were injected intraperitoneally with $30 \mathrm{ml}$ of $3 \%$ protease peptone in saline, followed by another injection of $10 \mathrm{ml} 24 \mathrm{~h}$ later. Two hours later, the peritoneal cavity was lavaged with $100 \mathrm{ml}$ of normal saline.

PMN were suspended in $0.34 \mathrm{M}$ sucrose and homogenized at $4^{\circ} \mathrm{C}$ with a homogenizer. The homogenate was centrifuged at 200 $\mathrm{g}$ for $10 \mathrm{~min}$ at $4^{\circ} \mathrm{C}$, and the supernatant was removed and centrifuged at $8700 \mathrm{~g}$ for $20 \mathrm{~min}$ at $4^{\circ} \mathrm{C}$. The pellet containing the mixed granule fraction was extracted with $0 \cdot 2 \mathrm{M}$ sodium acetate buffer $\mathrm{pH} 4.0$ containing $10 \mathrm{mM} \mathrm{CaCl}_{2}$, sonicated and then centrifuged at $100000 \mathrm{~g}$ for $1 \mathrm{~h}$ at $4^{\circ} \mathrm{C}$. The supernatant fluid contained granule protein [10]. The granule proteins were chromatographed on an FPLC mono S cation-exchange column, HR5/5 (Pharmacia, Uppsala, Sweden). Bound proteins were eluted with a gradient from 0.15 to $2.0 \mathrm{~mol} / 1 \mathrm{NaCl}$ in $0.05 \mathrm{~mol} / \mathrm{l}$ sodium acetate buffer, $\mathrm{pH} 4.0$ at a flow rate of $1 \mathrm{ml} / \mathrm{minute}$. The peaks between 0.6 and $0.8 \mathrm{~mol} / 1 \mathrm{NaCl}$ contained MPO and elastase. The combined and concentrated fractions of these peaks were applied to a FPLC Superose 12 gel filtration column HR16/50 (Pharmacia). Protein was eluted with $0.05 \mathrm{~mol} / \mathrm{l}$ sodium acetate buffer pH 5.0 at a flow rate of $0.5 \mathrm{ml} /$ minute and resulted in only 2 major peaks. The peak corresponding to a molecular weight of approximately $140 \mathrm{kDa}$ was identified as MPO by enzymatic activity and by ELISA [11].

\section{Rat MPO ELISA}

Microtitre plates were coated overnight with rat MPO at $5 \mu \mathrm{g} / \mathrm{ml}$ in phosphate buffered saline (PBS). After blocking with PBS containing $2 \%$ bovine serum albumin (BSA, Bioatlantic, Nantes, France), test sera were incubated either undiluted for one monoclonal mouse anti-rat MPO supernatant, or diluted in PBS containing $0 \cdot 1 \%$ Tween for 4 purified mouse anti-human MPO monoclonal antibody $(1 \mu \mathrm{g} / \mathrm{ml}), 7$ normal mice sera $(1 \mu \mathrm{g} / \mathrm{ml}$ final IgG concentration), 36 MPO-ANCA positive human sera and 10 negative control human sera $(1: 50)$. Binding was detected with either alkaline phosphatase-conjugated anti-mouse Ig (A8438, Sigma, St Louis, MO, USA) diluted 1:30 000, or alkaline phosphatase-conjugated anti-human IgG (A3150, Sigma) $1: 1000$, before addition of substrate. All incubations were done using $100 \mu \mathrm{l} /$ well at $37^{\circ} \mathrm{C}$ for one hour, with triple washes in PBS-Tween $0 \cdot 1 \%$. Positivity was defined as values greater than 3 SD over the mean of the negative mouse or human controls.

\section{Human MPO assays}

MPO-ANCA was determined using commercial kits according to the manufacturer's instructions (MK031, The binding site, Birmingham, UK) in 36 consecutive ANCA positive samples from 36 patients, both during active disease and at follow-up. Mouse anti-human MPO monoclonal reagents were characterized by ELISA and Western blotting as previously reported, using purified human MPO (Calbiochem, La Jolla, CA, USA) [12]. Three $\operatorname{IgG}(1 \mathrm{~B} 10,2 \mathrm{C} 7,2 \mathrm{~F} 2)$ and one $\operatorname{IgA}(1 \mathrm{H} 2)$ monoclonal reagents, that recognized distinct epitopes on human MPO [12] were used.

Mouse anti-rat MPO supernatant was incubated undiluted, and binding was compared to seven normal mouse sera as described for rat MPO ELISA.
Inhibition of human or mouse anti-MPO reagents binding to rat $M P O$ with human MPO

Serial dilution curves were used to determine the limiting rat MPO coating concentration in the rat MPO ELISA, that is the concentration below which a decrease binding of mouse anti-rat MPO antibody is observed in the assay.

Serial dilution of MPO-ANCA positive sera or mouse antihuman MPO monoclonal that exhibited significant binding in the rat MPO ELISA were applied in this rat MPO ELISA using limiting antigen coating concentration. The limiting antibody concentration was defined as the serum dilution below which a decrease binding in this ELISA was observed. Both limiting antigen coating concentration and limiting antibody concentrations were used for subsequent inhibition studies.

Anti-human MPO reagents were preincubated with purified human MPO (Calbiochem, $2 \mu \mathrm{g} / \mathrm{ml}$ to $0 \cdot 12 \mu \mathrm{g} / \mathrm{ml}$ ) or an irrelevant protein (BSA $2 \mu \mathrm{g} / \mathrm{ml}$ ) in glass tubes for one hour at $37^{\circ} \mathrm{C}$, before incubation on rat MPO coated ELISA plates. Results were expressed as the percentage of inhibition calculated according to the following formula:

$$
\% \text { inhibition }=\frac{1-(\mathrm{S} / \mathrm{MPO}-\mathrm{C})}{\mathrm{S}-\mathrm{C}} \times 100
$$

Where $\mathrm{S}=$ binding of test serum, $\mathrm{S} / \mathrm{MPO}=$ binding of test serum after preincubation with human MPO and $\mathrm{C}=$ binding of control sera.

\section{Binding of human MPO-ANCA on rat MPO ELISA after} adsorption of non-specific anti-rat activity

A chromatography column was filled with $1 \mathrm{~g}$ of $\mathrm{CNBr}$ activated sepharose (17-0430-01, Pharmacia), and washed in $200 \mathrm{ml} 1 \mathrm{mM}$ $\mathrm{HCl}$. One $\mathrm{ml}$ of pooled normal rat sera was added diluted $1: 4$ in coupling buffer. Remaining sepharose active groups were blocked with $0 \cdot 1 \mathrm{M}$ Tris- $\mathrm{HCl} \mathrm{pH} 8$ for $2 \mathrm{~h}$ at room temperature. Human MPO-ANCA positive sera were passed down the column and the unbound fraction was collected. IgG concentrations were checked, and binding of native and adsorbed sera were compared at the same final $\mathrm{IgG}$ concentration in rat MPO ELISA.

Mouse IgA anti-human MPO monoclonal binding to human or rat $M P O$ in the presence of jacalin

Jacalin is a lectin known to bind to the sugar residues of the IgA hinge region, responsible for IgA stickiness in solid phase assays. Mouse IgA anti-MPO monoclonal was preincubated with $25 \mu \mathrm{g} /$ $\mathrm{ml}$ jacalin (AbCys, Paris, France) for $1 \mathrm{~h}$ at room temperature. Inhibition of binding in rat MPO ELISA was expressed as:

$\frac{\text { [binding without lectin - binding with lectin] }}{\text { binding without lectin }} \times 100 \%$

\section{RESULTS}

Binding of human MPO-ANCA and mouse anti-human MPO monoclonal to rat $M P O$

Only one of 36 human MPO-ANCA positive sera $\left(\mathrm{N}^{\circ} 4\right)$ exhibited significant binding in rat MPO ELISA (3.6 SD over the mean binding of 10 control human sera).

The three mouse $\operatorname{IgG}$ anti-human MPO monoclonal did not recognize rat MPO. Only one mouse IgA anti-human MPO monoclonal bound to rat MPO ( $85 \mathrm{SD}$ over the mean binding of seven 


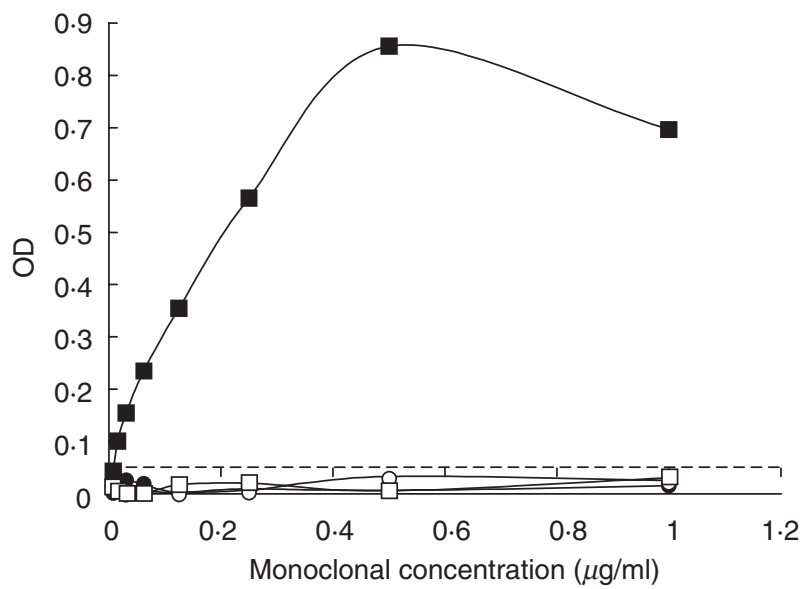

Fig. 1. Serial dilution curves of four mouse anti-human MPO monoclonal reagents in rat MPO ELISA. Only the mouse IgA anti-human MPO monoclonal $(1 \mathrm{H} 2, \mathbf{\square})$ bound to rat MPO. None of the mouse IgG antihuman MPO monoclonals $(2 \mathrm{C} 7, \bullet ; 1 \mathrm{~B} 10, \mathrm{O} ; 2 \mathrm{~F} 2, \square)$ exhibited binding to rat MPO. The dotted line is the threshold for positivity in the assay (3 SD above the mean of 7 control mouse sera).

normal mouse sera) (Fig. 1), with binding of the positive control (mouse anti-rat MPO supernatant) of $137.76 \mathrm{SD}$. The avidity of human polyclonal MPO-ANCA and mouse anti-human or rat MPO monoclonal should not be compared since controls and species specific reagents are different.

\section{Binding of human MPO-ANCA and mouse anti-rat MPO monoclonal to human $M P O$}

Human MPO-ANCA activity ranged from 11 to $>100 \mathrm{U}$ (mean $62 \cdot 2 \pm 36 \cdot 85 \mathrm{SD}$ ), with positivity over $9 \mathrm{U}$. Serum from patient $\mathrm{N}^{\circ} 4$ exhibited MPO-ANCA > $100 \mathrm{U}$, and was drawn during apparent clinical remission in 1996. Patient no. 4 presented on March 1994 with pneumonia, rapidly progressive glomerulonephritis and purpura. He was diagnosed as microscopic polyangiitis and treated by steroids and cyclophosphamide boluses. He is in remission since 1995 despite persisting MPO-ANCA activity.

The mouse anti-rat MPO monoclonal did not bind human MPO.

\section{Inhibition of binding to rat MPO by preincubation with human MPO}

The binding of the human MPO-ANCA positive serum to rat MPO was poorly inhibited by preincubation with human MPO in the liquid phase (maximum $26 \%$ at $0 \cdot 12 \mu \mathrm{g} / \mathrm{ml}$ ) (Fig. 2). The binding of the mouse $\operatorname{IgA}$ anti-human MPO monoclonal was only inhibited by preincubation with high concentration of human MPO (maximum $35 \%$ at $2 \mu \mathrm{g} / \mathrm{ml}$ ), suggesting that this IgA molecule may bind to rat MPO by non antigen specific sites (Fig. 2). The binding of mouse anti-rat MPO monoclonal reagent to rat MPO was also poorly inhibited by preincubation with human MPO (maximum $21 \%$ at $0 \cdot 12 \mu \mathrm{g} / \mathrm{ml}$ ) (Fig. 2).

\section{Inhibition of mouse IgA anti-human MPO monoclonal binding} to rat $M P O$ by jacalin

Jacalin did not prevent the binding of the mouse anti-human MPO monoclonal to rat MPO or human MPO $(0 \%$ inhibition).

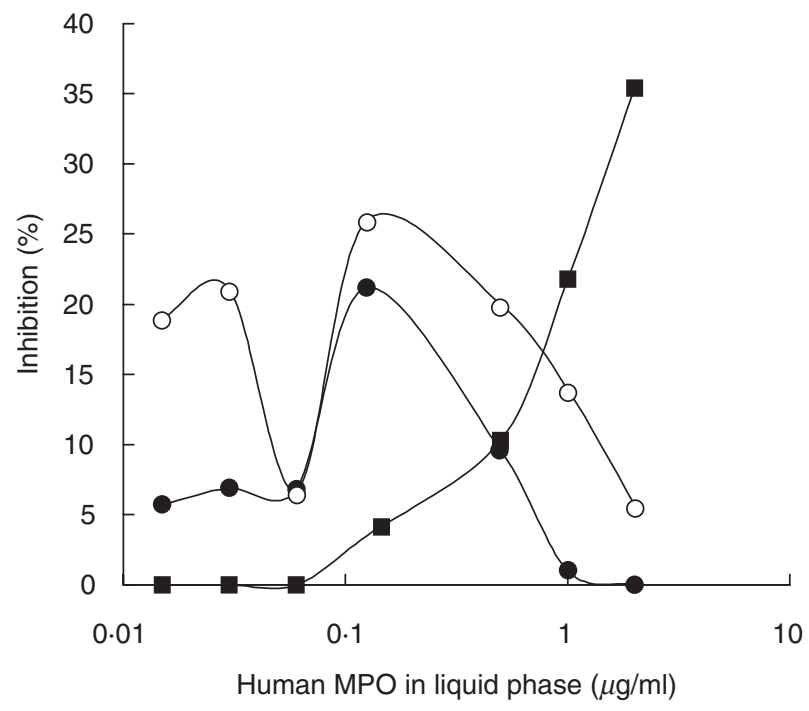

Fig. 2. Inhibition of binding to rat MPO by preincubation with human MPO in the liquid phase. Both limiting rat MPO coating concentration and limiting antibody concentrations were used in this assay (see methods). Antibodies (, anti-rat MPO monoclonal; $\mathbf{\square}, 1 \mathrm{H} 2$ anti-human MPO monoclonal; $\bigcirc$, the only human serum exhibiting binding in the rat MPO ELISA) were preincubated with serial dilution of human MPO $(2 \mu \mathrm{g} / \mathrm{ml}$ to $0 \cdot 12 \mu \mathrm{g} / \mathrm{ml}$ ) or an irrelevant protein (BSA $2 \mu \mathrm{g} / \mathrm{ml}$ ) in the liquid phase before coating in this rat MPO ELISA. Inhibition of binding is expressed as percentage.

Inhibition of human MPO-ANCA positive serum binding to rat MPO after adsorption of non-specific anti-rat activity

The binding of this human MPO-ANCA positive serum to rat MPO was not affected by the passage through the chromatography column containing normal rat proteins.

\section{DISCUSSION}

Our study show that most human MPO-ANCA and all our mouse anti-human MPO IgG monoclonal reagents recognize antigenic determinants on human MPO which are absent on rat MPO. Inversely, the mouse anti-rat MPO monoclonal does not bind to human MPO, confirming that rat MPO epitopes have been altered during species evolution. MPO is a key enzyme involved in the generation of powerful oxygen species, that are crucial for the protection against attack by foreign microorganisms. This may explain the high sequence homology of rodent (mouse) and human peroxidases through species evolution [13]. Therefore, it is striking that human auto-antibodies bind to epitopes which recently appeared after evolution from low mammals to human. The reason why tolerance to those newly appeared epitopes is less accurate remains to be determined. Negative selection in the thymus is mediated by random presentation of self epitopes. Those newly appeared determinants may escape to the screen of the maturing immune system.

Most epitopes recognized by anti-human MPO mice monoclonal reagents are not present on rat MPO. Therefore, mice injected with human MPO preferentially develop antibodies against xeno-epitopes which are not present in rodents. This does not rule out the possibility that rat injected with human MPO may develop some cross-reactivity against rat MPO, as previously 
shown [6,7]. However, our study points out that human MPO presents several epitopes which are not present in rodents. We therefore suggest that rat MPO would be a better antigen with which to induce models of vasculitis in rats. However, certain models of renal vasculitis in the rat appear to be induced by [14] or exacerbated by immunization with human MPO [6-9], and MPO/ anti-MPO complexes has not been consistently observed [10], raising the possibility that induction of ANCA by human MPO may have pathological consequences.

Two new strategies have been used to generate auto-antibodies directed to ANCA auto-antigens. First, the tolerance to ANCA auto-antigens was broken in BN rats by injection of syngenic apoptotic neutrophils [15]. However, those animals did not exhibit signs of vasculitis. Second, anti-MPO antibodies were raised by injection of mouse MPO to MPO deficient mice, and adoptive transfer of their splenocytes in recombinase activating gene 2 deficient mice (RAG-/-) led to crescentic glomerulonephritis with immune complex deposition and MPO-ANCA activity [16]. Furthermore, susceptible strains of mice may spontaneously develop crescentic glomerulonephritis in the presence of ANCA, and this model has already been used to compare new therapeutic strategies [17]. However, several additional autoantibodies are detected in these mice, and not all of them develop glomerulonephritis [18]. The search for an animal model that could be manipulated to develop systemic vasculitis with antibodies directed to neutrophil cytoplasmic auto-antigens remains a challenge.

The inability to induce anti-MPO-mediated vasculitis in rodents based on the passive transfer of human anti-MPO antibodies has been in part attributed to the lack of reactivity of antihuman MPO to the rodent antigen. This contention has however, never been verified experimentally. In fact, the high degree of homology between the human and rodent molecules [13] would predict some cross-reactivity of the antibodies. The lack of crossreativity of anti-human MPO to the rat antigen, despite the high degree of homology between the two molecules suggests that these antibodies are directed against epitopes that are not shared. A comparison of the amino acid sequences of the two homologueous molecules may help elucidate the major antigenic epitopes of the MPO molecule.

\section{REFERENCES}

1 Falk RJ, Jennette JC. ANCA small-vessel vasculitis. J Am Soc Nephrol 1997; 8:314-22.

2 Boomsma MM, Stegeman CA, van der Leij MJ et al. Prediction of relapses in Wegener's granulomatosis by measurement of antineutrophil cytoplasmic antibody levels: a prospective study. Arthritis Rheum 2000; 43:2025-33.
3 Falk RJ, Terrell RS, Charles LA, Jennette JC. Anti-neutrophil cytoplasmic autoantibodies induce neutrophils to degranulate and produce oxygen radicals in vitro. Proc Natl Acad Sci USA 1990; 87:4115-9.

4 Savage COS, Pottinger BE, Gaskin G, Pusey CD, Pearson JD. Autoantibodies developing to myeloperoxidase and proteinase 3 in systemic vasculitis stimulate neutrophil cytotoxicity toward cultured endothelial cells. Am J Pathol 1992; 141:335-42.

5 Esnault VLM, Mathieson PW, Thiru S, Oliveira DBG, Lockwood CM. Autoantibodies to myeloperoxidase in Brown Norway rats treated with mercuric chloride. Laboratory Invest 1992; 67:114-20.

6 Brouwer E, Huitema MG, Klok PA et al. Antimyeloperoxidaseassociated proliferative glomerulonephritis: an animal model. J Exp Med 1993; 177:905-14.

7 Brouwer E, Klok PA, Huitema MG, Weening JJ, Kallenberg CGM. Renal ischemia/reperfusion injury contributes to renal damage in experimental anti-myeloperoxidase-associated proliferative glomerulonephritis. Kidney Int 1995; 47:1121-9.

8 Heeringa P, Brouwer E, Klok PA et al. Autoantibodies to myeloperoxidase aggravate mild anti-glomerular-basement-membrane mediated glomerular injury in the rat. Am J Pathol 1996; 149:1695-706.

9 Heeringa P, Foucher P, Klok PA et al. Systemic injection of products of activated neutrophils and $\mathrm{H}_{2} \mathrm{O}_{2}$ in myeloperoxidase-immunized rats leads to necrotizing vasculitis in the lungs and gut. Am J Pathol 1997; 151:131-40.

10 Yang JJ, Jennette JC, Falk RJ. Immune complex glomerulonephritis is induced in rats immunized with heterologous myeloperoxidase. Clin Exp Immunol 1994; 97:466-73.

11 Yang JJ, Preston GA, Pendergraft WF et al. Internalization of proteinase 3 is concomitant with endothelial cell apoptosis and internalization of myeloperoxidase with generation of intracellular oxidants. Am J Pathol 2001; 158:581-92.

12 Audrain MAP, Baranger TAR, Moguilevski N et al. Anti-native and recombinant myeloperoxidase monoclonals and human autoantibodies. Clin Exp Immunol 1997; 107:127-13.

13 Venturelli D, Shirsat N, Gemperlein I, Bittenbender S, Hudson S, Rovera G. Nucleotide sequence of cDNA for murine myeloperoxidase. Nucl Acids Res 1989; 17:5852.

14 Smyth CL, Smith J, Cook HT, Haskard DO, Pusey CD. Immunisation with MPO directly induces small vessel vasculitis with pauci-immune focal segmental glomerulonephritis and alveolar haemorrhage in WKY rats. J Am Soc Nephrol 2002; 13:170A.

15 Patry YC, Trewick DC, Gregoire M et al. Rats injected with syngenic rat apoptotic neutrophils develop antineutrophil cytoplasmic antibodies. J Am Soc Nephrol 2001; 12:1764-8.

16 Xiao H, Heeringa P, Hu P et al. Antineutrophil cytoplasmic autoantibodies specific for myeloperoxidase cause glomerulonephritis and vasculitis in mice. J Clin Invest 2002; 110:955-63.

17 Birck R, Newman M, Back W, Nemoto K, Yard B, van der Woude FJ. 15-deoxyspergualin and cyclophosphamide, but not mycophenolate mofetil, prolong survival and attenuate renal disease in SCG/Kj mice. J Am Soc Nephrol 2001; 12:627A.

18 Kinjoh K, Kyoguru M, Good RA. Genetic selection for crescent formation yields mouse strain with rapidly progressive glomerulonephritis and small vessel vasculitis. Proc Natl Acad Sci USA 1993; 90:3413-7. 